\title{
Coalgebraic Logic and Synthesis of Mealy Machines
}

\author{
M.M. Bonsangue ${ }^{1,2}$, Jan Rutten ${ }^{2,3, \star}$, and Alexandra Silva ${ }^{2, \star \star}$ \\ ${ }^{1}$ LIACS - Leiden University \\ ${ }^{2}$ Centrum voor Wiskunde en Informatica (CWI) \\ ${ }^{3}$ Vrije Universiteit Amsterdam (VUA)
}

\begin{abstract}
We present a novel coalgebraic logic for deterministic Mealy machines that is sound, complete and expressive w.r.t. bisimulation. Every finite Mealy machine corresponds to a finite formula in the language. For the converse, we give a compositional synthesis algorithm which transforms every formula into a finite Mealy machine whose behaviour is exactly the set of causal functions satisfying the formula.
\end{abstract}

\section{Introduction}

A Mealy machine $(S, f)$ consists of a set $S$ of states and a transition function $f: S \rightarrow$ $(B \times S)^{A}$ assigning to each state $s \in S$ and input symbol $a \in A$ a pair $\left\langle b, s^{\prime}\right\rangle$, consisting of an output symbol $b \in B$ and a next state $s^{\prime} \in S$. Typically one writes

$$
f(s)(a)=\left\langle b, s^{\prime}\right\rangle \Longleftrightarrow s \stackrel{a \mid b}{\longrightarrow} s^{\prime}
$$

One of the most important applications of Mealy machines is their use in the specification of sequential digital circuits. Taking binary inputs and outputs, there is a wellknown correspondence between such binary Mealy machines, on the one hand, and sequential digital circuits built out of logical gates and some kind of memory elements, on the other. In present day text books on logic design [11] — on the construction of sequential digital circuits - Mealy machines are still the most important mathematically exact means for the specification of the intended behaviour of circuits.

There does not seem to exist, however, a generally accepted way of formally specifying Mealy machines themselves. The only formal approach we are aware of is the general model for categories with feedback in [6], which can be instantiated to Mealy machines. However, Mealy machines are typically "defined" in a natural language such as English. This obviously leads to ambiguities, inconsistencies and plain errors [4].

In this paper, we propose a simple but adequate and expressive logical language for the specification of Mealy machines. Here adequate means that the logical equivalence corresponds to a natural behavioural equivalence on Mealy machines, whereas expressive means that every finite Mealy machine can be represented by a (finite) formula. Finally, simple means that the logic contains precisely what is needed to obtain this

\footnotetext{
* Partially supported by EU project IST-33826 CREDO (http: / / credo. cwi .nl).

** Partially supported by the Fundação para a Ciência e a Tecnologia, Portugal, under grant number SFRH/BD/27482/2006.
} 
goal, and nothing more. The latter point is an important distinguishing factor in comparison with some already existing formalisms in the literature, discussed below.

Briefly stated, our approach is coalgebraic. Mealy machines are a basic and wellunderstood family of coalgebras, of the functor $M(S)=(B \times S)^{A}$. The crucial coalgebraic insight is that the properties of Mealy machines (coalgebras) are fully dictated by (the shape of) their defining functor $M$. This has led, for instance, to the identification of a final Mealy machine, in [14], as the set of all causal stream functions from $A^{\omega}$ to $B^{\omega}$.

Following coalgebraic methodology, we apply general insights from coalgebraic modal logic (see e.g. [122]) and define a logic whose basic operations derive directly from the functor $M$. The equivalence induced by the logic coincides with that induced by the functor $M$. Further, the logic comes equipped with a proof system for reasoning about universal validity that we prove sound and complete.

All finite Mealy machines can be specified as a formula in the logic. The main technical contribution of the paper is the construction, for every formula in the logic, of a finite Mealy Machine whose behaviour is exactly characterised by the formula.

\subsection{Related Work}

Automata synthesis is a popular and very active research area [13|8|4|15|5]. Most of the work done on synthesis has as main goal to find a proper and sufficiently expressive type of automata to encode a specific type of logic (such as LTL [15] or $\mu$-calculus [8]).

Technically, the synthesis from a $\mu$-calculus formula $\varphi$ consists in translating $\varphi$ into an alternating automaton $\mathcal{A}_{\varphi}$, reducing $\mathcal{A}_{\varphi}$ into a non-deterministic automaton which is then checked for non-emptiness [8]. The same process has been recently generalized to $F$-coalgebras in [10]. In this paper, we use a different approach. We construct a deterministic Mealy machine for a formula directly, by considering the formula as a state of the automaton containing enough information about its successors.

Although Mealy machines are in one-to-one correspondence with sequential digital circuits, not much work has been done for their specification and synthesis. In [6], an algebra for systems with feedback is given, but no synthesis is presented. In [15], a compositional algorithm for synthesizing Generalized Mealy machines (GMMs) from LTL formulae is presented. GMMs are a special class of non-deterministic Mealy machines that have the acceptance condition of generalized Büchi automata. In this paper, we will remain in the world of deterministic Mealy machines, the one corresponding to sequential digital circuits. Moreover, our work exploits the structure of the Mealy machine and, therefore, the resulting logic is simpler than LTL (but expressive enough for deterministic Mealy machines).

The logic most similar to ours is the one presented in [4]. There a logic for formal specification of hardware protocols is presented, and an algorithm for the synthesis of a Mealy machine is given. Their logic corresponds to the conjunctive fragment of LTL. Their synthesis process is standard: first a non-deterministic Büchi automaton is synthesized, secondly a powerset construction is used to make the automaton deterministic and, finally, the propositions on the states are used to determine the inputs and outputs for each state of the Mealy machine. Because of our coalgebraic approach, the equivalence induced by our logic is canonical, and the logic comes with a proof system that 
is sound and complete. Further, our synthesis process remains within standard Mealy machines and the behaviour of the synthesized automata is exactly characterized by the original formula. Apart from [14[5], where synthesis for a special case of 2-adic arithmetic is treated, we did not find any other work on the direct synthesis of deterministic Mealy machines. From these papers we inherit the basic coalgebraic approach, that we use here to derive our expressive logical specification language for Mealy machines.

In summary, the work presented in this paper distinguishes itself from all existing work as follows. Our specification logic is derived directly from the functor, which results in a very simple and consistent logic that has exactly the operators needed to fully specify Mealy machines. Note that being simple does not mean this logic has less expressive power than others. In the context of applications (such as circuits logic design), this logic has all the relevant operators.

\section{Mealy Machines}

We give the basic definitions on Mealy machines and introduce the notions of simulation and bisimulation.

First we recall the following definition. A (bounded) meet-semilattice is a set $B$ equipped with a binary operation $\wedge_{B}$ and a constant $\top_{B} \in B$, such that $\wedge_{B}$ is commutative, associative and idempotent. The element $T_{B}$ is neutral w.r.t. $\wedge_{B}$. As usual, $\wedge_{B}$ gives rise to a partial ordering $\leq_{B}$ on the elements of $B$ :

$$
b_{1} \leq_{B} b_{2} \Leftrightarrow b_{1} \wedge_{B} b_{2}=b_{1}
$$

Every set $S$ can be transformed into a meet-semilattice by taking the collection $\mathcal{P} S$ of all subsets of $S$ with intersection as meet. We use semilattices to represent data structures equipped with an information order: $b_{1} \leq_{B} b_{2}$ means that $b_{1}$ is more concrete than $b_{2}$.

Our running examples will all use the four element meet-semilattice:

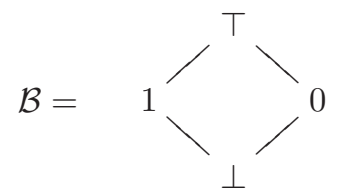

Here, the $T$ element is used for abstracting (under-specification) from any concrete data; the $\perp$ element denotes inconsistency (over-specification) of information; and the elements 0 and 1 are concrete output values.

Now let $A$ be a finite set and let $B$ be a (possibly infinite) meet-semilattice. A Mealy machine $(S, f)$ with inputs in $A$ and outputs in $B$ consists of a set of states $S$ together with a function

$$
f: S \rightarrow(B \times S)^{A}
$$

For a given state $s \in S$ and an input $a \in A$, the function $f$ returns a pair $f(s)(a)=$ $\left\langle b, s^{\prime}\right\rangle$, consisting of an output value $b \in B$ and a state $s^{\prime} \in S$. Typically we will write

$$
f(s)(a)=\left\langle s[a], s_{a}\right\rangle
$$


and call $s[a]$ the (initial) output on input $a$ and $s_{a}$ the next state on input $a$. We shall also use the following convention for the representation of Mealy machines:

$$
f(s)(a)=\left\langle b, s^{\prime}\right\rangle \Longleftrightarrow s \stackrel{a \mid b}{\longrightarrow} s^{\prime}
$$

In coalgebraic terms, a Mealy machine $(S, f)$ is a coalgebra of the functor $M:$ Set $\rightarrow$ Set defined, for any set $X$, as $M(X)=(B \times X)^{A}$.

A homomorphism from a Mealy machine $(S, f)$ to a Mealy machine $(T, g)$ is a function $h: S \rightarrow T$ preserving initial outputs and next states:

$$
h(s)[a]=s[a] \text { and } h\left(s_{a}\right)=h(s)_{a}
$$

(which is equivalent to the condition that $g \circ h=M(h) \circ f$, where the functor $M$ is defined on functions as usual).

Machines where $A$ is the two-element set $\{0,1\}$ and $B$ is the meet-semilattice $\mathcal{B}$ are called binary, and they are fully specified if only 0 or 1 are used as output elements (and never $\perp$ or $\top$ ).

For an example, consider the following binary Mealy machine with $S=\left\{s_{1}, s_{2}\right\}$ and the transition function defined by the following picture.

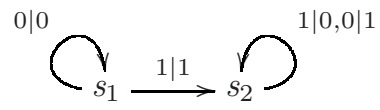

This machine computes the two's complement of a given binary number.

Next we define the notion of simulation, which can be used to obtain abstraction, and bisimulation, which plays an important role in the minimization of Mealy machines.

Definition 1 ((Bi)simulation for Mealy). Let $(S, f)$ and $(T, g)$ be two Mealy machines. We call a relation $R \subseteq S \times T$ a simulation if for all $(s, t) \in S \times T$ and $a \in A$

$$
s R t \Rightarrow\left(s[a] \leq_{B} t[a] \text { and } s_{a} R t_{a}\right)
$$

We call $R$ a bisimulation relation if both $R$ and its (relational) inverse $R^{-1}$ are simulations.

We write $s \lesssim t$ (resp. $s \sim t$ ) whenever there exists a simulation relation (bisimulation relation) containing $(s, t)$; and we call $\lesssim$ and $\sim$ the similarity and bisimilarity relations. By definition, we have $\lesssim \cap \lesssim^{-1}=\sim$.

As an example, consider the following two binary Mealy machines:

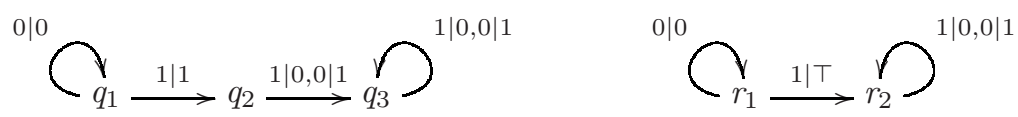

Observe that $q_{3}$ and $q_{2}$ are bisimilar, since $R=\left\{\left(q_{2}, q_{3}\right),\left(q_{3}, q_{3}\right)\right\}$ is a bisimulation. A minimal machine is obtained by identifying all bisimilar states, yielding our two's complement machine above. 
Now, note that the rightmost machine can be simulated by any fully specified binary machine substituting either 0 or 1 as output for the abstract $T$ value in the transition from $r_{1}$ to $r_{2}$. For example, considering the above two's complement machine, we have $s_{1} \lesssim r_{1}$ because $S=\left\{\left(s_{1}, r_{1}\right),\left(s_{2}, r_{2}\right)\right\}$ is a simulation relation.

Next we recall the construction of a final Mealy machine with inputs in $A$ and outputs in $B$. Finality plays an important role in minimization as well as in the proof system (in Section 3).

Let $A^{\omega}=\{\sigma \mid \sigma:\{0,1,2, \ldots\} \rightarrow A\}$, the set of all infinite streams over $A$. For $a \in A$ and $\sigma \in A^{\omega}$, we define:

$$
a: \sigma=(a, \sigma(0), \sigma(1), \sigma(2), \ldots) \quad \sigma^{\prime}=(\sigma(1), \sigma(2), \sigma(3), \ldots)
$$

We call a function $f: A^{\omega} \rightarrow B^{\omega}$ causal if for all $\sigma \in A^{\omega}$ and $n \geq 0$, the $n$th output value $f(\sigma)(n)$ depends only on the first $n$ input values $(\sigma(0), \ldots, \sigma(n-1))$. Let

$$
\Gamma=\left\{f: A^{\omega} \rightarrow B^{\omega} \mid f \text { is causal }\right\}
$$

The set $\Gamma$ can be turned into a Mealy machine $(\Gamma, \gamma)$ by defining $\gamma(f)(a)=\left\langle f[a], f_{a}\right\rangle$ as follows:

$$
f[a]=f(a: \sigma)(0) \text { (where } \sigma \text { is arbitrary) } \quad f_{a}(\sigma)=(f(a: \sigma))^{\prime}
$$

(Note that by causality the value of $f(a: \sigma)(0)$ depends only on $a$.) The following theorem is a minor variation on [14, Prop.2.3 and Corr.2.3].

Theorem 2 (Finality of $(\Gamma, \gamma)$ ). For every Mealy machine $(S, f)$ there exists a unique homomorphism $h: S \rightarrow \Gamma$. It satisfies, for all $s, s^{\prime} \in S$ :

$$
s \lesssim s^{\prime} \Longleftrightarrow h(s) \lesssim h\left(s^{\prime}\right)
$$

where on $\Gamma$, similarity coincides with the elementwise ordering induced by $B$ :

$$
f \lesssim g \Longleftrightarrow \forall \sigma \in A^{\omega} \forall n \geq 0 . f(\sigma)(n) \leq_{B} g(\sigma)(n)
$$

Since the identity function is always a homomorphism, bisimilarity is equality on $\Gamma$. As a consequence, the image $h(S)$ of a Mealy machine $S$ is in fact its minimisation with respect to bisimilarity.

\section{Mealy Logic}

We present a logic for Mealy machines and define its semantics and a satisfaction relation.

Definition 3 (Mealy formulae). Let $A$ be a set of input actions and let $B$ be a meetsemilattice of output actions. Furthermore, let $X$ be a set of (recursion or) fixed point variables. The set $L$ of Mealy formulae $i$ given by the following BNF syntax. For $a \in A$, $b \in B$, and $x \in X$ :

$$
\phi::=t t|x| a(\phi)|a \downarrow b| \phi \wedge \phi \mid \nu x . \psi
$$


where $\psi \in L_{g}$, the set of guarded formulae, which is given by:

$$
\psi::=t t|a(\phi)| a \downarrow b|\psi \wedge \psi| \nu x . \psi
$$

We call $a(\phi)$ a transition formula and $a \downarrow b$ an output formula. Note that our language does not include disjunction or negation. As we will discuss in 3.2 this is a natural restriction and does not decrease the expressiveness of our logic. Moreover, in the same section we will also point out the reasons for only having one type of fixed point operator. Also note that for every unguarded Mealy formula there exists an equivalent guarded formula, as a consequence of [9, Theorem 2.1].

The modal fragment of our logic (i.e, the set of closed formulae without the $\nu$ operator) is a special case of the coalgebraic logic obtained by a Stone-type duality [1]2].

In what follows, we shall concentrate on the set $L_{g}^{c}$ of formulae that are both guarded and closed, that is, without free occurrences of fixed point variables $x$. We turn the set $L_{g}^{c}$ into a Mealy machine (coalgebra)

$$
\lambda: L_{g}^{c} \rightarrow\left(B \times L_{g}^{c}\right)^{A}
$$

by defining $\lambda$ as follows. For $a \in A$ and $\phi \in L_{g}^{c}$, we write $\lambda(\phi)=\left\langle\phi[a], \phi_{a}\right\rangle$ and we define $\phi[a]$ and $\phi_{a}$ by

$$
\begin{aligned}
& t t[a] \quad=\top_{B} \quad t t_{a}=t t
\end{aligned}
$$

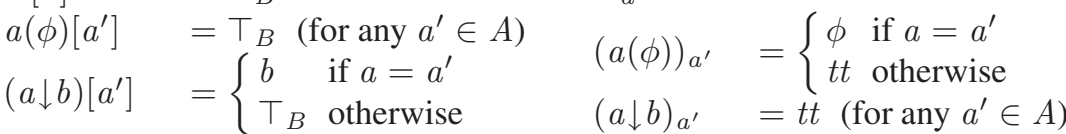

$$
\begin{aligned}
& \left(\phi_{1} \wedge \phi_{2}\right)[a]=\phi_{1}[a] \wedge \phi_{B} \phi_{2}[a] \quad\left(\phi_{1} \wedge \phi_{2}\right)_{a}=\left(\phi_{1}\right)_{a} \wedge\left(\phi_{2}\right)_{a} \\
& (\nu x . \psi)[a]=(\psi[\nu x . \psi / x])[a] \quad(\nu x . \psi)_{a}=(\psi[\nu x . \psi / x])_{a}
\end{aligned}
$$

Here, $\psi[\nu x . \psi / x]$ denotes syntactic substitution, replacing in $\psi$ every free occurrence of $x$ by $\nu x . \psi$.

The above definition uses induction on the following complexity measure, which is based on the number of nested unguarded occurrences of $\nu$-formulae:

$$
\begin{aligned}
& N(t t) \quad=N(a \downarrow b)=N(a(\phi))=0 \\
& N\left(\phi_{1} \wedge \phi_{2}\right)=\max \left\{N\left(\phi_{1}\right), N\left(\phi_{2}\right)\right\}+1 \\
& N(\nu x . \psi)=1+N(\psi)
\end{aligned}
$$

In order to see that the definition of $\phi[a]$ and $\phi_{a}$ is well-formed, note that in the case of $\nu x . \psi$, we have:

$$
N(\psi)=N(\psi[\nu x \cdot \psi / x])
$$

This can easily be proved by (standard) induction on the syntactic structure of $\psi$, since $\psi$ is guarded (in $x$ ).

Note that the (sub)machine generated by a formula $\phi \in L_{g}^{c}$ by repeatedly applying $\lambda$ will in general be infinite. In Section 4 an algorithm to produce a finite Mealy machine from a formula $\phi \in L_{g}^{c}$ will be presented. 
Having a Mealy coalgebra structure on $L_{g}^{c}$ has two advantages. First, it provides us, by finality of $\Gamma$, directly with a natural semantics because of the existence of a (unique) homomorphism:

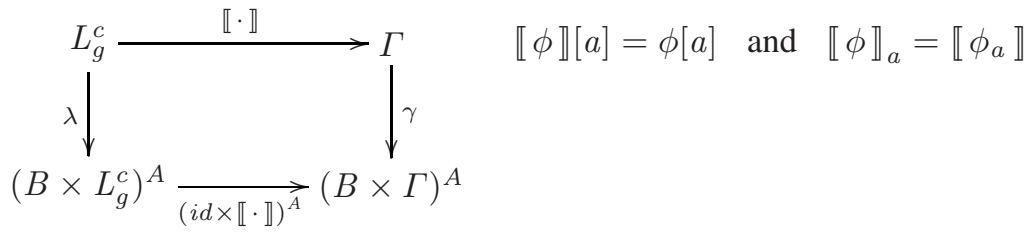

It assigns to every formula $\phi$ a causal stream function $\llbracket \phi \rrbracket: A^{\omega} \rightarrow B^{\omega}$.

The second advantage of the Mealy structure on $L_{g}^{c}$ is that it lets us use the notion of Mealy simulation to define when a state $s \in S$ of a Mealy machine $(S, f)$ satisfies a formula $\phi \in L_{g}^{c}$, by defining:

$$
s \models \phi \quad \Leftrightarrow \quad s \lesssim \phi
$$

For brevity, we say that a Mealy machine $(S, f)$ satisfies a formula $\phi$ if some state in $S$ satisfies $\phi$.

Proving satisfaction then amounts to the construction of a simulation relation $R \subseteq$ $S \times L_{g}^{c}$ between $(S, f)$ and $(L, \lambda)$ such that $s R \phi$.

The above definition is equivalent to the following, more classical definition of satisfaction. For every valuation $\eta$ : $\operatorname{Var} \rightarrow \mathcal{P}(S)$, we define a satisfaction relation $\models_{\eta}$, by induction, as follows:

$$
\begin{array}{ll}
s \models_{\eta} t t & \text { for all } s \\
s \models_{\eta} a(\phi) & \text { iff } s_{a} \models_{\eta} \phi \\
s \models_{\eta} a \downarrow b & \text { iff } s[a] \leq_{B} b \\
s \models_{\eta} \phi_{1} \wedge \phi_{2} & \text { iff } s=_{\eta} \phi_{1} \text { and } s \models_{\eta} \phi_{2} \\
s \models_{\eta} x & \text { iff } s \in \eta(x) \\
s \models_{\eta} \nu v . \psi & \text { iff } \exists T \subseteq S . s \in T \text { and } \forall t \in T . t \models_{\eta[T / v]} \psi
\end{array}
$$

Here, $\eta[T / v]$ denotes the valuation such that, for every $x \in \operatorname{Var}$, with $x \neq v$, returns $\eta(x)$ and for $x=v$ returns $T$.

Note that in this definition single occurrences of $x \in X$ are allowed. It can be shown, by a fairly straightforward and not very instructive proof, that the two definitions of satisfaction are equivalent. More precisely, if $\emptyset$ denotes the everywhere empty valuation, we have:

$$
s \lesssim \phi \Leftrightarrow s \models \emptyset \phi
$$

for every $\phi \in L_{g}^{c}$. We omit the proof and will work in what follows with the definition of satisfaction as simulation.

The following theorem shows that our logic is sufficiently expressive to characterise bisimilarity. 


\section{Theorem 4}

(1) For all states $s, s^{\prime}$ of a Mealy machine $(S, f)$,

$$
s \sim s^{\prime} \quad \text { iff } \quad \forall \phi \in L_{g}^{c} . s \models \phi \Leftrightarrow s^{\prime} \models \phi
$$

(2) If $S$ is finite then there exists for any $s \in S$ a formula $\phi_{s} \in L_{g}^{c}$ such that

$$
\forall s^{\prime} \in S . s \sim s^{\prime} \quad \text { iff } \quad s^{\prime} \models \phi_{s}
$$

Proof. (1) Because $s \sim s^{\prime}$ implies $s \lesssim s^{\prime}$ and $s^{\prime} \lesssim s$ we have, for any $\phi \in L_{g}^{c}$,

$$
s=\phi \Longleftrightarrow s \lesssim \phi \Longleftrightarrow s^{\prime} \lesssim \phi \Longleftrightarrow s^{\prime} \models \phi
$$

For the converse, note, for any $s \in S, a \in A$, and $\phi \in L_{g}^{c}$, that $s \models a \downarrow s[a]$ and

$$
s_{a} \models \phi \Longleftrightarrow s_{a} \lesssim \phi \Longleftrightarrow s \lesssim a(\phi) \Longleftrightarrow s \models a(\phi)
$$

As a consequence, the following relation

$$
R=\left\{\left\langle s, s^{\prime}\right\rangle \in S \times S \mid \forall \phi \in L_{g}^{c} . s \models \phi \Leftrightarrow s^{\prime} \models \phi\right\}
$$

and its inverse $R^{-1}$ are simulation relations on $S$. Thus $R$ is a bisimulation.

(2) It is sufficient to construct for a given $s \in S$ a formula $\phi_{s}$ with $s \sim \phi_{s}$. To this end, we associate with every state $s \in S$ a variable $x_{s} \in X$ and a formula $\phi_{s}=\nu x_{s} . \psi_{s}$ defined by

$$
\psi_{s}=\bigwedge_{a \in A} a\left(x_{s_{a}}\right) \wedge a \downarrow s[a]
$$

Syntactically replacing free occurrences of $x_{s^{\prime}}$ by $\phi_{s^{\prime}}$ in $\phi_{s}\left(s \neq s^{\prime}\right)$ will ensure that all $\phi_{s}$ will be in $L_{g}^{c}$. By construction, $s \sim \phi_{s}$.

Let us illustrate the last construction above. Recall the two's complement Mealy machine presented before:

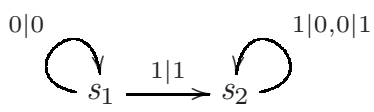

We define $\phi_{1}=\nu x_{1} \cdot \psi_{1}$ and $\phi_{2}=\nu x_{2} \cdot \psi_{2}$ by

$$
\psi_{1}=0\left(x_{1}\right) \wedge 0 \downarrow 0 \wedge 1\left(x_{2}\right) \wedge 1 \downarrow 1 \quad \psi_{2}=0\left(x_{2}\right) \wedge 0 \downarrow 1 \wedge 1\left(x_{2}\right) \wedge 1 \downarrow 0
$$

Substituting $\phi_{2}$ for $x_{2}$ in $\psi_{1}$ then yields

$$
\phi_{1}=\nu x_{1} .0\left(x_{1}\right) \wedge 0 \downarrow 0 \wedge 1\left(\phi_{2}\right) \wedge 1 \downarrow 1 \quad \phi_{2}=\nu x_{2} .0\left(x_{2}\right) \wedge 0 \downarrow 1 \wedge 1\left(x_{2}\right) \wedge 1 \downarrow 0
$$

By construction we have $s_{1} \sim \phi_{1}$ and $s_{2} \sim \phi_{2}$.

\subsection{Proof System}

We now introduce a proof system for assertions of the form $\phi_{1} \leq \phi_{2}$, where $\leq$ is the relation of logical entailment between the closed formulae $\phi_{1}$ and $\phi_{2}$. 


$$
\begin{aligned}
& \text { (refl) } \quad \phi \leq \phi \\
& (\wedge-e 1) \phi_{1} \wedge \phi_{2} \leq \phi_{1} \\
& (\text { top }) \quad \phi \leq t t \\
& \text { (trans) } \frac{\phi_{1} \leq \phi_{2} \quad \phi_{2} \leq \phi_{3}}{\phi_{1} \leq \phi_{3}} \\
& (\wedge-e 2) \quad \phi_{1} \wedge \phi_{2} \leq \phi_{2} \\
& (\wedge-i) \quad \frac{\phi \leq \phi_{1} \quad \phi \leq \phi_{2}}{\phi \leq \phi_{1} \wedge \phi_{2}} \\
& (a \downarrow-\top) t t \leq a \downarrow \top_{B} \\
& (a()-\top) t t \leq a(t t) \\
& (a \downarrow-\wedge) a \downarrow b_{1} \wedge a \downarrow b_{2} \leq a \downarrow\left(b_{1} \wedge_{B} b_{2}\right)(a()-\wedge) a\left(\bar{\phi}_{1}\right) \wedge a\left(\phi_{2}\right) \leq a\left(\phi_{1} \wedge \phi_{2}\right) \\
& (a \downarrow-\leq) \frac{b_{1} \leq_{B} b_{2}}{a \downarrow b_{1} \leq a \downarrow b_{2}} \\
& (a()-\leq) \frac{\phi_{1} \leq \phi_{2}}{a\left(\phi_{1}\right) \leq a\left(\phi_{2}\right)} \\
& (\nu-i) \quad \frac{\phi \leq \psi[\phi / x]}{\phi \leq \nu x . \psi} \\
& (\nu-e) \quad \frac{\psi[\nu x . \psi / x] \leq \phi}{\nu x . \psi \leq \phi}
\end{aligned}
$$

The first group of axioms and rules gives to the set of formulae the structure of a meetsemilattice. Further, there are axioms and rules for the two modal operators, showing the interactions between the transition and output formulae with the meet-semilattice structure. Finally, the last two rules $(\nu-i)$ and $(\nu-e)$ can be explained as stating that the term $\nu x . \psi$ is the greatest postfixed point, when viewing the formula $\psi$ as a (monotone) map on formulae.

We write $\vdash \phi_{1} \leq \phi_{2}$ to indicate that the assertion $\phi_{1} \leq \phi_{2}$ is derivable from the above axioms and rules. Note that the converse of $(a \downarrow-\wedge)$ is derivable from $(a \downarrow-\leq)$ and $(\wedge-i)$. Similarly, also the converses of $(a \downarrow-\top),(a()-\top)$ and $(a()-\wedge)$ are derivable.

Theorem 5 (Soundness). The above proof system is sound, that is, for closed formulae $\phi_{1}$ and $\phi_{2}, \vdash \phi_{1} \leq \phi_{2}$ implies that for all Mealy machines $(S, f)$ and $s \in S$ if $s \models \phi_{1}$ then $s=\phi_{2}$.

Proof. By induction on the length of proofs.

Next we turn to the completeness for the modal fragment $L_{m}$ of our Mealy logic $L$, where a modal formula is a formula with neither fixed point operators nor variables. Note that the (Lindenbaum algebra of) $L_{m}$ is a meet-semilattice.

Let $\Theta$ be the set of all filters of (the Lindenbaum algebra of) $L_{m}$, where a filter of a meet-semilattice is a non-empty upper closed subset $\mathcal{F}$ such that if $a, b \in \mathcal{F}$ then also $a \wedge b \in \mathcal{F}$. The set $\Theta$ can be turned into a Mealy machine $(\Theta, \theta)$ by defining, for $F \in \Theta$ and $a \in A, \theta(F)(a)=\left\langle F[a], F_{a}\right\rangle$, where

$$
F[a]=\bigwedge\{b \mid a \downarrow b \in F\} \quad F_{a}=\{\phi \mid a(\phi) \in F\} .
$$

Note that in order for $F[a]$ to be well defined we assume $B$ to be a finite meet-semilattice. In case $B$ is infinite, we would need $B$ to be a complete meet-semilattice.

Theorem 6. For every Mealy machine $(S, f)$ there exists a unique homomorphism $k_{S}: S \rightarrow \Theta$. In particular, the homomorphism $k_{\Gamma}: \Gamma \rightarrow \Theta$ is an isomorphism.

As a consequence of Theorem 4 , the isomorphism $k_{\Gamma}: \Gamma \rightarrow \Theta$ is also an order isomorphism, where the order on $\Theta$ is subset inclusion. The logical significance of the 
above result is that a finitary logic with only finite conjunctions suffices to completely describe all Mealy machines up to bisimilarity. In fact the modal fragment of our logic is a special case of coalgebraic logic obtained by a Stone-type duality [1|2].

Theorem 6 together with the next lemma gives a logical interpretation of the final coalgebra: its elements correspond to canonical models (in the logical sense) of the Mealy logic.

Lemma 7. For every modal formula $\phi$ and filter $F \in \Theta, F \models \phi$ if and only if $\phi \in F$.

Proof. By induction on the structure of $\phi$, using the fact that $F$ is a filter and the above definition of $\theta: \Theta \rightarrow(B \times \Theta)^{A}$.

We can finally prove the completeness of the modal fragment of our Mealy logic.

Theorem 8 (Completeness). For modal formulae $\phi_{1}$ and $\phi_{2}$, if $s \models \phi_{1}$ implies $s \models \phi_{2}$ for all Mealy machines $(S, f)$ and $s \in S$, then $\vdash \phi_{1} \leq \phi_{2}$.

Proof. Assume $\forall \phi_{1} \leq \phi_{2}$. It is enough to find a state $s$ in a Mealy machine $(S, f)$ such that $s=\phi_{1}$ but $s \not \models \phi_{2}$. Define $F_{\phi_{1}}=\left\{\psi \mid \phi_{1} \leq \psi\right\}$. It is not very difficult to verify that $F_{\phi_{1}}$ is a filter, hence it is an element of $\Theta$. Clearly, $\phi_{1} \in F_{\phi_{1}}$ but, by our assumption $\phi_{2} \notin F_{\phi_{1}}$. We can now conclude by applying Lemma 7

\subsection{Adding Negation}

The logic we have considered so far contains no negation. Extending the logic with negated formulae is not problematic as long as we consider Mealy machines with outputs in a Boolean algebra $B$ (like the two-element set). In this case, we can still turn the set of (possibly negated) formulae into a Mealy coalgebra by extending our definition of $\lambda$ at the beginning of section 3 with

$$
(\neg \phi)[a]=\neg B(\phi[a]) \quad(\neg \phi)_{a}=\neg(\phi)_{a} .
$$

It is easy to see that according to this definition negation distributes up to bisimulation over conjunction (de Morgan law), and over the modal operators (a sign that the machine is indeed deterministic). Further, negation is classical, meaning that $\neg(\neg \phi) \sim \phi$. Clearly, disjunctions and $\mu$-recursive formulae can be defined as derived operators.

From the logical point of view, this means that the Lindenbaum algebra of the resulting logic with negation is the free Boolean algebra over the meet-semilattice of the Mealy logic we considered here. In this case one can apply the isomorphism $\operatorname{UFilt}(B(L)) \cong$ Filt $(L)$ to obtain analogous soundness and completeness results as above, where $L$ is a meet-semilattice, $B(L)$ is the free Boolean algebra over $L$ and $\operatorname{UFilt}(B(L))$ is the set of ultrafilters of $B(L)$.

\section{Synthesis}

We will now describe the synthesis process that produces a Mealy machine from an arbitrary (closed and guarded) Mealy formula 1 . Each state of the resulting Mealy machine will be a formula constructed in such a way that if $s$ is the state corresponding to

\footnotetext{
${ }^{1}$ The source code in HASKELL can be downloaded from www. cwi . nl/ ams /mealy
} 
a formula $\phi$, then $s \sim \phi$. This implies that the semantics of $s$ is exactly the set of causal functions satisfying $\phi$.

\subsection{Formulae Normalization}

We have seen that the first group of six axioms and rules of our proof system gives to the set of formulae the structure of a meet-semilattice. In order to guarantee the termination of the synthesis process we will need to identify formulae that are provably equivalent using only these axioms and rules. For instance, the formulae

$$
a(t t) \wedge a \downarrow b \wedge t t \wedge a \downarrow b \text { and } a(t t) \wedge a \downarrow b
$$

are equivalent.

To normalize a formula $\phi$, we need to eliminate any redundancy present in the formula: in a conjunction, $t t$ can be eliminated and, by idempotency, the conjunction of two syntactically equivalent formulae can be simplified.

The function norm encodes this procedure. We define it by induction on the formula structure as follows:

$$
\begin{array}{ll}
\operatorname{norm}(t t) & =t t \\
\operatorname{norm}(a(\phi)) & =a(\operatorname{norm}(\phi)) \\
\operatorname{norm}(a \downarrow b) & =a \downarrow b \\
\operatorname{norm}\left(\phi_{1} \wedge \phi_{2}\right) & =\operatorname{conj}\left(\operatorname{rem}\left(\operatorname{flatten}\left(\operatorname{norm}\left(\phi_{1}\right) \wedge \operatorname{norm}\left(\phi_{2}\right)\right)\right)\right) \\
\operatorname{norm}(\nu x . \phi) & =\nu x \cdot(\operatorname{norm}(\phi)) .
\end{array}
$$

Here, conj takes a list of formulae $\left[\phi_{1}, \ldots, \phi_{n}\right]$ and returns the formula $\phi_{1} \wedge \ldots \wedge \phi_{n}$ (conj applied to the empty list yields $t$ ), rem removes duplicates in a list and flatten takes a formula $\phi$ and produces a list of formulae by omitting brackets and replacing $\wedge$-symbols by commas:

$$
\begin{aligned}
& \text { flatten }\left(\phi_{1} \wedge \phi_{2}\right)=\operatorname{flatten}\left(\phi_{1}\right) \cdot \operatorname{flatten}\left(\phi_{2}\right) \\
& \text { flatten }(t) \quad=[] \\
& \operatorname{flatten}(\phi) \quad=[\phi], \phi \in\left\{a \downarrow b, a\left(\phi_{1}\right), \nu x . \phi_{1}\right\}
\end{aligned}
$$

In this definition, - denotes list concatenation and $[\phi]$ the singleton list containing $\phi$. Note that an occurrence of $t t$ in a conjunction is eliminated because flatten $(t)=[]$.

For example, the normalization of the two formulae above will result in the same formula $-a(t t) \wedge a \downarrow b$.

Note that norm still distinguishes the formulae $\phi_{1} \wedge \phi_{2}$ and $\phi_{2} \wedge \phi_{1}$. For simplifying the presentation of the normalization algorithm, we decided not to identify these formulae, since this will not influence termination. However, in the implementation, in order to reduce the number of states, those formulae are identified. In the examples below this situation will never occur.

\subsection{Synthesis}

We first describe what happens in a single step of the synthesis process.

The function $\delta$, which does one-step synthesis for a single formula, takes a formula $\phi \in L_{g}^{c}$ and produces a partial Mealy machine. Below, $\delta$ will be used in the function $\Delta$, which synthesises the total Mealy machine. 
The function $\delta$ is defined, by induction on the complexity measure $N$ defined in Section 3, as follows:

$$
\begin{aligned}
& \delta(t t)(a)=\left\langle\top_{B}, t t\right\rangle \\
& \delta\left(a^{\prime}(\phi)\right)(a)= \begin{cases}\left\langle\top_{B}, \text { norm }(\phi)\right\rangle & a=a^{\prime} \\
\left\langle\top_{B}, t t\right\rangle & \text { otherwise } \\
\langle b, t t\rangle & a=a^{\prime} \\
\left\langle\top_{B}, t t\right\rangle & \text { otherwise }\end{cases} \\
& \delta\left(a^{\prime} \downarrow b\right)(a)=\delta\left(\phi_{1}\right)(a) \sqcap \delta\left(\phi_{2}\right)(a) \\
& \delta\left(\phi_{1} \wedge \phi_{2}\right)(a)=\left\langle b, \text { norm }\left(\phi^{\prime}\right)\right\rangle \text { where }\left\langle b, \phi^{\prime}\right\rangle=\delta(\phi[\nu x . \phi / x])(a) \\
& \delta(\nu x . \phi)(a)=
\end{aligned}
$$

where $\sqcap$ is defined as: $\left\langle b_{1}, \phi_{1}\right\rangle \sqcap\left\langle b_{2}, \phi_{2}\right\rangle=\left\langle b_{1} \wedge_{B} b_{2}, \operatorname{norm}\left(\phi_{1} \wedge \phi_{2}\right)\right\rangle$.

Note that this function is very similar to the function $\lambda$ presented in Section 3 . In fact, the difference is the normalization that is now being applied to the formulae so that a finite machine will be produced.

As an example, consider the formula $\phi=1 \downarrow 0 \wedge(\nu x .1(x))$, specifying a binary Mealy machine. We can easily compute that $\delta(\phi)(0)=\left\langle\top_{B}, t t\right\rangle$ and

$$
\begin{aligned}
\delta(\phi)(1) & =\delta(1 \downarrow 0)(1) \sqcap \delta(\nu x .1(x))(1) \\
& =\langle 0, t t\rangle \sqcap\left\langle\top_{B}, \nu x .1(x)\right\rangle \\
& =\langle 0, \nu x .1(x)\rangle
\end{aligned}
$$

So, $\delta(\phi)$ is a (partial) finite function represented by the following diagram.

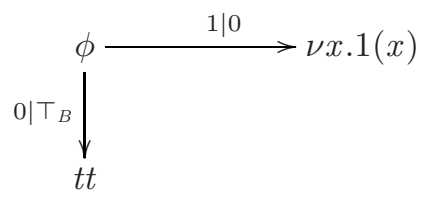

To compute the entire Mealy machine that satisfies $\phi$, we need to apply $\delta$ to the new states generated at each step repeatedly until all states in the automata have their transitions/outputs fully defined.

We implement this procedure with the auxiliary function $D$. The arguments of this function are two sets of states: sts $\subseteq L_{g}^{c}$, the states that still need to be processed and vis $\subseteq L_{g}^{c}$, the states that already have been visited (synthesized). For each $\phi \in s t s, D$ computes $\delta(\phi)$ and produces an intermediate transition function (possibly partial) by taking the union of all those $\delta(\phi)$. Then, it collects all new states appearing in this step and recursively computes the transition function for those.

$$
\begin{aligned}
& D(\text { sts }, \text { vis })= \begin{cases}\emptyset & \text { sts }=\emptyset \\
\text { trans } \cup D\left(\text { newsts, vis }{ }^{\prime}\right) & \text { otherwise }\end{cases} \\
& \text { where trans }=\{\langle\phi, \delta(\phi)\rangle \mid \phi \in \text { sts }\} \\
& \text { sts }^{\prime}=\text { collectStates }(\text { trans }) \\
& v_{i s^{\prime}}=\text { sts } \cup \text { vis } \\
& \text { newsts }=\text { sts }{ }^{\prime} \backslash v i s^{\prime}
\end{aligned}
$$

The function $\Delta$ takes a Mealy formula $\phi \in L_{g}^{c}$ and returns a Mealy machine that satisfies $\phi$ : 


$$
\Delta(\phi)=(\operatorname{dom}(f), f) \text { where } f=D(\{\operatorname{norm}(\phi)\}, \emptyset)
$$

The function dom returns the domain of a finite function.

Due to lack of space, the proof of finiteness and termination of the synthesis algorithm is not included. They are included in the extended version of this paper [3].

Let us look at an example. For the formula $\phi$ presented above $\Delta(\phi)=(S, f)$, where $S=\{t t, \phi, \nu x .1(x)\}$ and $f$ is represented by the following diagram.

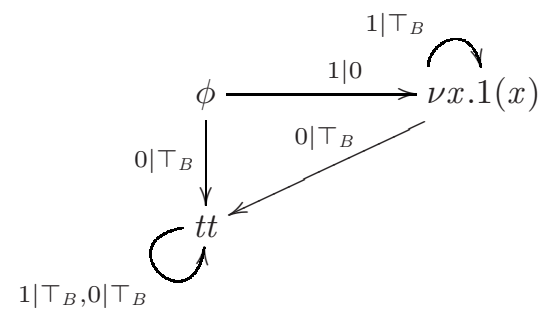

Note that the Mealy machine produced by $\Delta$ is not minimal. In this example, the states $t t$ and $\nu x .1(x)$ are bisimilar and could be identified.

The (special) output value $T_{B}$ allows us to define underspecified machines: if a given formula does not contain information about the output value for a given input $a$, then we do not return as output a concrete value but instead $\top_{B}$. If $\top_{B}$ is replaced by any other element $b \in B$ the resulting machine will still satisfy $\phi$.

Let us see a few other examples of the synthesis process. To simplify the presentation, we consider again binary machines and, moreover, the formulae presented below will only have information for the input 1 . Therefore, for the 0 input $\delta$ will always return $\left\langle T_{B}, t t\right\rangle$.

Let us start with $\phi_{1}=1(1 \downarrow 0) \wedge(\nu x .1(x))$. We have:

$$
\begin{aligned}
\delta\left(\phi_{1}\right)(1) & =\delta(1(1 \downarrow 0))(1) \sqcap \delta(\nu x .1(x))(1) \\
& =\left\langle T_{B}, 1 \downarrow 0\right\rangle \sqcap\left\langle\top_{B}, \nu x .1(x)\right\rangle \\
& =\left\langle\top_{B}, 1 \downarrow 0 \wedge(\nu x .1(x))\right\rangle
\end{aligned}
$$

We now repeat the process for $1 \downarrow 0 \wedge(\nu x .1(x))$, which will yield $\delta(1 \downarrow 0 \wedge(\nu x .1(x)))$ $(1)=\langle 0, \nu x .1(x)\rangle$. Finally, we calculate $\delta(\nu x .1(x))(1)=\left\langle\top_{B}, \nu x .1(x)\right\rangle$.

The complete Mealy machine is represented in the following diagram:

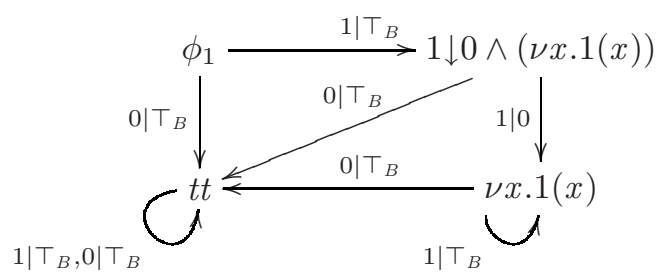

Now, take $\phi_{2}=\nu x .1(1 \downarrow 0) \wedge 1(x)$. Because $1(1 \downarrow 0)$ has no $x$ 's one could be tempted to assume that the automaton for $\phi_{2}$ would be the same as the one for $\phi_{1}$. However, that is not the case. The synthesis algorithm will produce the following automaton for $\phi_{2}$. 


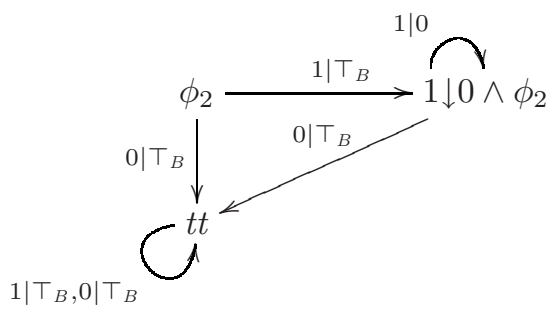

As a last example, let $\phi_{3}=\nu x .1(x \wedge(\nu y .1(y) \wedge 1 \downarrow 0))$. We have:

$$
\begin{aligned}
\delta\left(\phi_{3}\right)(1) & =\delta\left(1\left(\phi_{3} \wedge(\nu y .1(y) \wedge 1 \downarrow 0)\right)\right)(1) \\
& =\left\langle\top_{B}, \phi_{3} \wedge(\nu y .1(y) \wedge 1 \downarrow 0)\right\rangle
\end{aligned}
$$

and

$$
\begin{aligned}
& \delta\left(\phi_{3} \wedge(\nu y .1(y) \wedge 1 \downarrow 0)\right)(1) \\
= & \delta\left(\phi_{3}\right)(1) \sqcap \delta(\nu y .1(y) \wedge 1 \downarrow 0)(1) \\
= & \left\langle\top_{B}, \phi_{3} \wedge(\nu y .1(y) \wedge 1 \downarrow 0)\right\rangle \sqcap\langle 0, \nu y .1(y) \wedge 1 \downarrow 0\rangle \\
= & \left\langle 0, \operatorname{norm}\left(\phi_{3} \wedge(\nu y .1(y) \wedge 1 \downarrow 0) \wedge(\nu y .1(y) \wedge 1 \downarrow 0)\right)\right\rangle \\
= & \left\langle 0, \phi_{3} \wedge(\nu y .1(y) \wedge 1 \downarrow 0)\right\rangle
\end{aligned}
$$

Note that if norm would not have been applied, the resulting state $\phi_{3} \wedge(\nu y .1(y) \wedge$ $1 \downarrow 0) \wedge(\nu y .1(y) \wedge 1 \downarrow 0)$ would be regarded as a new state, even though it is equivalent to $\phi_{3} \wedge(\nu y .1(y) \wedge 1 \downarrow 0)$. Moreover, applying $\delta$ to this state (for input 1 ) would yield again an equivalent but (syntactically) different state, namely $\phi_{3} \wedge(\nu y .1(y) \wedge 1 \downarrow 0) \wedge$ $(\nu y .1(y) \wedge 1 \downarrow 0) \wedge(\nu y .1(y) \wedge 1 \downarrow 0)$. This illustrates that the function $\lambda$, defined in Section 3 , generally produces an infinite machine. However, the identifications made by norm ensure the termination of the synthesis process.

\section{Conclusions and Future Work}

We have given a coalgebraic account of Mealy machines and provided a logical specification language for them. Despite its simplicity, the logic is expressive in the sense that all Mealy machines can be characterized by finite formulae, but also in the sense that logical equivalence corresponds to bisimulation. Further, the logic is sound and the modal fragment complete for all Mealy machines.

The specification language is finitary and includes a fixed point operator.

Other temporal operators can be defined as derived operators. Interestingly, the language is already expressive enough to characterize all Mealy machines even without negation and disjunction. Even stronger, for binary Mealy machines the addition of negation does not increase the expressive power of the logic. This situation is typical also of deterministic finite automata: the addition of negation in regular expressions does not increase the class of languages that they characterize, even though regular languages are closed under complement.

Our main result is an algorithm for the synthesis of a Mealy machine from a formula. Our synthesis algorithm is compositional, in the sense that the semantics of the 
Mealy machine synthesized from a formula can be obtained by suitably composing the semantics of the Mealy machines synthesized from sub-formulae.

In this paper we have explored the synthesis of one particular type of automata, the Mealy machines. With a small variation of the logic one can easily obtain a similar result for Moore automata. More generally, different type of automata can be obtained by varying the functor under consideration on the category of sets. It would be interesting to generalize the present result in order to synthesize coalgebras for different functors.

Acknowledgements. We would like to thank Clemens Kupke, Helle Hvid Hansen and Yde Venema for valuable suggestions and discussions.

\section{References}

1. Bonsangue, M.M., Kurz, A.: Duality for logics of transition systems. In: Sassone, V. (ed.) FOSSACS 2005. LNCS, vol. 3441, pp. 455-469. Springer, Heidelberg (2005)

2. Bonsangue, M.M., Kurz, A.: Presenting functors by operations and equations. In: Aceto, L., Ingólfsdóttir, A. (eds.) FOSSACS 2006. LNCS, vol. 3921, pp. 172-186. Springer, Heidelberg (2006)

3. Bonsangue, M.M., Rutten, J.J. M.M., Silva, A.: Coalgebraic Logic and Synthesis of Mealy Machines. CWI Technical report R0705 (2007)

4. Clarke, E.M., German, S.M., Lu, Y., Veith, H., Wang, D.: Executable protocol specification in esl. In: Johnson, S.D., Hunt Jr., W.A. (eds.) FMCAD 2000. LNCS, vol. 1954, pp. 197-216. Springer, Heidelberg (2000)

5. Hansen, H.H., Costa, D., Rutten, J.J.M.M.: Synthesis of mealy machines using derivatives. ENTCS 164(1), 27-45 (2006)

6. Katis, P., Sabadini, N., Walters, R.F.C.: Feedback, trace and fixed-point semantics. ITA 36(2), 181-194 (2002)

7. Kozen, D.: Results on the propositional $\mu$-calculus. TCS 27, 333-354 (1983)

8. Kupferman, O., Vardi, M.: $\mu$-calculus synthesis. In: Nielsen, M., Rovan, B. (eds.) MFCS 2000. LNCS, vol. 1893, pp. 497-507. Springer, Heidelberg (2000)

9. Kupferman, O., Vardi, M., Wolper, P.: An automata-theoretic approach to branching-time model checking. J. ACM 47(2), 312-360 (2000)

10. Kupke, C., Venema, Y.: Coalgebraic automata theory: basic results. Technical Report SENE0701, CWI, The Netherlands (2007)

11. Marcovitz, A.B.: Introduction to Logic Design. McGraw-Hill, New York (2005)

12. Moss, L.: Coalgebraic logic. Annals of Pure and Applied Logic 96 (1999)

13. Pnueli, A., Rosner, R.: On the synthesis of a reactive module. In: POPL 1989, pp. 179-190 (1989)

14. Rutten, J.J.M.M.: Algebraic specification and coalgebraic synthesis of mealy automata. ENTCS 160, 305-319 (2006)

15. Tini, S., Maggiolo-Schettini, A.: Compositional synthesis of generalized mealy machines. Fundam. Inform. 60(1-4), 367-382 (2004) 